\title{
L'éducation des adultes comme stratégie d'implantation de l'éducation permanente en milieu universitaire: la leçon de la France
}

\author{
MARIO FERLAND*
}

\section{RÉSUMÉ}

Le concept d'éducation permanente suppose la mise en place d'un système éducatif entièrement nouveau. Pour les universités, cela entraîne une transformation profonde, déjà amorcée par l'éducation des adultes; aussi apparaît-il normal que celle-ci soit utilisée comme stratégie d'implantation de l'éducation permanente en milieu universitaire. Les approches privilégiées diffèrent d'une institution à l'autre, mais elles se ramènent à trois grandes formules, selon que les structures mises en place pour l'éducation des adultes sont intégrées, parallèles ou périphériques.

Dans son effort pour amener les universités à l'éducation permanente, le gouvernement français a opté systématiquement pour la formule périphérique. Une enquête menée récemment dans $76 \%$ des universités françaises permet de dégager certaines lignes de force susceptibles de servir de leçons pour l'ensemble des universités: (a) au plan politique comme au plan conceptuel, il existe, entre l'éducation des adultes et l'éducation permanente, une différence de niveaux dont il faut toujours tenir compte; (b) le développement de l'éducation des adultes requiert une certaine autonomie; (c) il n'existe pas de monopole institutionnel universitaire en éducation des adultes; (d) l'approche périphérique tend à favoriser la marginalisation de l'éducation des adultes.

En somme, il y a tout lieu de croire que l'éducation des adultes ne constitue pas la seule, ni même la meilleure stratégie d'implantation de l'éducation permanente: l'éducation des jeunes pourrait, à cet effet, s'avérer plus efficace.

\begin{abstract}
The idea of continuing education implies a complete restructuration of the educational system. The university, for one, will have to undergo profound changes, some of which have actually taken place through adult education: hence the propensity to use adult education as a strategy for implementing continuing education in the university. Tactical means used differ from one institution to the other, but they can be grouped into three major categories, according to the organizational structure of adult education: the integrated, the parallel and the peripheral approach.
\end{abstract}

* Direction générale du premier cycle, Université Laval 
In its effort to convert the universities to continuing education, the French government has systematically adopted the peripheral approach. A recent survey made in $76 \%$ of the French universities has revealed some trends, in the treatment of adult and continuing education, from which lessons can be drawn: (a) politically as well as ideologically, adult and continuing education are different and one must always take that difference into account; (b) the development of adult education as a university function requires a certain amount of autonomy; (c) the university has no institutional monopoly in adult education; (d) the peripheral approach tends to promote the marginalization of adult education.

All in all, it seems that adult education is not the unique, nor even the best strategy for implementing continuing education at the university level: this could probably be more efficiently achieved through the education of youngsters.

Il existe, entre les concepts d'éducation permanente et d'éducation des adultes, une filiation certaine qui relève davantage de l'histoire que de la sémiologie. L'éducation des adultes, qui a pris naissance et s'est développée, comme activité organisée, au cours du $\mathrm{XIXe}$ siècle, a fait prendre conscience de l'importance et de la capacité pour tout être humain de se développer (ou de s'éduquer) tout au long de sa vie, sur des plans divers et par des moyens divers: le concept de l'éducation permanente est donc issu, en quelque sorte historiquement, de celui de l'éducation des adultes.

Mais ces deux concepts n'en sont pas "parents" pour autant. Il est vrai que si l'éducation est permanente, elle inclut l'éducation des jeunes et celle des adultes; toutefois, comme on se plaît souvent à le souligner, l'éducation permanente n'est pas l'éducation des adultes. Et pourtant, que de fois n'a-t-on pas vu ces deux expressions utilisées indifféremment l'une à la place de l'autre et, encore, par des spécialistes en la matière, tant la filiation historique soulignée plus haut a pu se transformer en une filiation sémiologique.

Mais il y a plus: le rapprochement entre les concepts d'éducation permanente et d'éducation des adultes a fini par prendre une valeur stratégique. En effet, en se basant sur le fait que le concept d'éducation des adultes a suscité celui d'éducation permanente dont il fait partie, on en est venu à faire en sorte que l'éducation des adultes devienne l'instrument privilégié d'un changement organisationnel vers l'éducation permanente.

Cette utilisation de l'éducation des adultes comme stratégie d'implantation de l'éducation permanente a été adoptée par l'ensemble des universités québécoises et des universités françaises selon des formules diverses et avec une efficacité qui reste encore à vérifier.

La présente étude a précisément pour objets de fournir un exemple important d'une telle utilisation de l'éducation des adultes, soit celui des universités françaises, et d'en dégager des leçons pour l'ensemble des universités. A cet effet, nous chercherons, dans un premier temps, à mesurer l'impact des concepts d'éducation permanente et d'éducation des adultes sur le développement des institutions universitaires et à décrire les trois formules d'utilisation de l'éducation des adultes comme stratégie d'implantation de l'éducation permanente, selon la théorie de G. Pineau (1975); puis nous verrons dans quelles circonstances et avec quels résultats les universités françaises ont privilégié l'une de ces formules, celle dite "périphérique", après quoi il nous sera possible de formuler les leçons que le milieu universitaire en général peut tirer de cette expérience. 


\section{LES UNIVERSITÉS FACE À L'ÉDUCATION PERMANENTE ET À L'ÉDUCATION DES ADULTES}

\section{L'éducation permanente}

Parmi toutes les définitions de l'éducation permanente qui existent, nous nous en tiendrons, dans la présente étude, à celle que propose le Conseil de l'Europe; c'est sur cette définition, en effet, que le Conseil des universités du Québec s'est appuyé pour établir les objectifs généraux de l'enseignement supérieur (Québec, 1973) et c'est également cette définition qui a inspiré les auteurs de la loi d'orientation des universités françaises (France, 1968). Selon le Conseil de l'Europe (1970), l'éducation permanente se définit comme suit:

Principe organisateur de toute éducation, elle implique un système complet, cohérent et intégré, offrant les moyens propres à répondre aux aspirations d'ordre éducatif et culturel de chaque individu et conformes à ses facultés. Elle est destinée à permettre à chacun de développer sa personnalité toute sa vie durant, par son travail ou par ses activités de loisir. Elle est également déterminée par les responsabilités que chaque individu a envers la société (p. 78).

Ce qui retient surtout l'attention, dans cette définition, c'est qu'elle met en évidence le caractère essentiel de l'éducation permanente: servir de principe de base à un système éducatis entièrement renouvelé, auquel participent désormais toutes les composantes de la société.

Un nouveau système éducatif. Dans l'expression "éducation permanente", on est généralement porté à ne retenir que l'idée de continuation dans le temps, c'est-à-dire le fait que l'éducation est un phénomène qui se prolonge tout au long de la vie. Mais cette conception de l'éducation est beaucoup plus vaste. Elle s'appuie, en effet, sur la conviction que l'homme doit et peut se développer constamment, et ce, non seulement à des âges divers, mais encore sur des plans divers (v.g. intellectuel, social, moral, physique, etc.), par des moyens divers (v.g. cours, études personnelles, lectures, voyages, travail, activités sociales, etc.) et dans des organismes divers (v.g. écoles, syndicats, groupes sociaux, famille, milieu de travail, église, etc.).

L'éducation n'est donc pas réservée à une seule période de la vie (la jeunesse), à un seul type d'institution (l'école), à une seule méthode (l'enseignement) et aux seuls sujets scolaires (les langues, les mathématiques, l'histoire, etc.); elle est, au contraire, une activité constante à laquelle participe chacun des organismes qui composent la société.

Aussi, lorsqu'on dit de l'éducation permanente qu'elle est le principe organisateur et intégrateur de tout le système éducatif, cela signifie, qu'en contribuant au développement continuel et intégral de l'individu, l'école, le milieu de travail, les syndicats, les groupes sociaux, etc. prennent place dans un vaste ensemble voué à l'éducation. En somme, c'est par l'éducation permanente que s'organise le système éducatif et c'est par l'éducation permanente que chaque organisme s'intègre à ce système.

Une université transformée. A titre d'institution d'enseignement, l'université assume, par sa nature même, certaines responsabilités dans le développement des individus; mais, dans un contexte d'éducation permanente, elle partage maintenant ces responsabilités avec les autres organismes qui composent la société. Vu sous cet angle, le principe de l'éducation permanente apparaît, selon les termes mêmes du Conseil des universités du Québec (1973), comme une "idée qui inspire et rationnalise le développement (...) de l'université (p. 2): celle-ci doit, en effet, s'adapter non seulement au système lui-même, mais encore aux besoins éducatifs nouveaux. 
Par rapport au système, l'université devra définir la place et le rôle qu'elle y tiendra, de même que le type de relations qu'il lui faudra entretenir avec les autres organismes. Par rapport aux besoins éducatifs, la transformation devra s'effectuer principalement: (a) sur l'enseignement (contenu des cours, méthodes pédagogiques, évaluation des connaissances, rôle du professeur, etc.) et (b) sur les modes de fonctionnement (information, recrutement des clientèles, extension des services dans le temps et dans l'espace, etc.).

Le Conseil des universités du Québec (1973) énumère les points d'impact de l'éducation permanente, mais il ne précise ni la nature ni le sens des modifications à apporter. L'ampleur de la tâche à accomplir montre cependant qu'on a raison de considérer l'éducation permanente comme un défi pour l'université; défi d'autant plus grand que, parallèlement à cette transformation vers l'éducation permanente, l'université doit aussi répondre aux besoins de formation des adultes.

\section{L'éducation des adultes}

Bien qu'elle fasse partie de l'éducation permanente, l'éducation des adultes, comme nous l'avons souligné plus haut, n'en est pas moins différente. Alors que l'éducation permanente est une conception globale de l'éducation qui agit comme principe organisateur du système éducatif tout entier, l'éducation des adultes, elle, se situe non pas au niveau des principes, mais à celui de l'action.

Elle se définit généralement comme l'ensemble des activités systématiquement organisées, ayant pour but de donner aux personnes qui ont déjà accédé au marché du travail (ou qui sont en mesure d'y accéder) les connaissances, les comportements et les attitudes nécessaires soit à l'accomplissement de leurs tâches professionnelles présentes ou futures, soit à leur développement personnel. Pour atteindre leur objectif, ces activités doivent être élaborées et réalisées selon des principes, des méthodes et des techniques particulières que l'on a tendance à considérer de plus en plus comme une science: l'andragogie. Quant à l'ensemble des activités offertes dans cette optique, il constitue ce qu'on appelle la "formation continue", par opposition à la "formation initiale" qui, elle, s'adresse aux jeunes qui n'ont pas l'expérience du marché du travail.

Des comportements nouveaux pour l'université. De même que l'adoption du principe de l'éducation permanente a exigé des universités qu'elles prennent de nouvelles orientations, ainsi le développement de l'éducation des adultes comme phénomène social $\mathrm{a}$, depuis quelques années, imposé aux universités la prise en charge d'une responsabilité spécifique: chercher à répondre aux besoins de formation d'une clientèle particulière, différente, à bien des points de vue, de la clientèle des jeunes à laquelle elles sont habituées. Pour assumer efficacement cette responsabilité, les universités doivent utiliser une approche pédagogique et administrative qui tienne compte des caractéristiques propres aux adultes et qui, de ce fait, se distingue nettement de celle qui prévaut pour l'ensemble de l'université.

Sur le plan pédagogique, il leur faut, par exemple: (a) identifier et préciser des besoins de formation souvent mal perçus ou mal exprimés, (b) traduire ces besoins en activités éducatives appropriées et (c) assurer la préparation adéquate des personnes-ressources.

Sur le plan administratif, la prise en charge de l'éducation des adultes oblige l'université à adopter des comportements nouveaux et inusités tels que: (a) concevoir et diffuser une forme de publicité adaptée à la clientèle, (b) négocier les activités éducatives demandées par des organismes ou des groupes et (c) développer des modes particuliers de gestion financière et dispenser les services appropriés en dehors des temps et des lieux habituels. 
63 L'éducation des adultes comme stratégie de l'éducation permanente en milieu universitaire:

Dans le domaine de l'éducation des adultes, les universités sont amenées, en somme, à adopter une démarche de type commerciale: prospection des besoins du "marché", conception d'un "produit" (l'activité éducative) conforme aux exigences de la clientèle, marketing, budgétisation équilibrée, etc. On peut le regretter, on peut déplorer ce côté peu universitaire de l'éducation des adultes, mais on ne voit pas comment il en serait autrement: la formation continue, en effet, n'a pas, pour les adultes, ce caractère d'importance ou de nécessité que la formation initiale peut avoir pour les jeunes: il y a toujours, ici, un public à conquérir.

\section{L'éducation des adultes au service de l'éducation permanente}

Des approches différentes. Sous l'influence de facteurs tels que la structure administrative de l'université, l'importance des clientèles adultes à servir, et, surtout, l'adhésion à la théorie "intégriste" ou "séparatiste" sur l'apprentissage des adultes (Houle, 1968), l'organisation de l'éducation des adultes en milieu universitaire a pris des formes variées. On peut déjà soupçonner cette diversité d'approches institutionnelles dans l'éventail des vocables utilisés: service, centre ou direction de l'éducation des adultes, des études à temps partiel ou des cours du soir, extension de l'enseignement universitaire, etc.

Malgré la diversité de leurs formes, les différentes structures organisationnelles mises en place par les universités pour assumer leur responsabilité en éducation des adultes peuvent, selon l'étude qu'en a faite Gaston Pineau (1975), être ramenées à trois grands modèles: la formule intégrée, la formule parallèle et la formule périphérique.

La première de ces formules "se caractérise par le rattachement institutionnel de l'éducation des adultes aux éléments déjà existants" (p. 131). Selon cette formule, il n'est fait aucune distinction sur le plan administratif ni sur le plan pédagogique entre les adultes et les étudiants réguliers; ainsi les procédures d'admission et d'inscription s'appliquent intégralement pour les adultes qui doivent, par ailleurs, s'accommoder des enseignements existants dont l'horaire a pu être adapté à leur disponibilité. La formule intégrée se veut, par conséquent, un ajustement de l'éducation des adultes aux modes de fonctionnement et aux exigences de l'ensemble de l'université.

La deuxième formule "consiste en la création d'organismes spécifiques d'éducation d'adultes complètement indépendants" (p. 132). Cette formule, dite "parallèle", s'appuye sur le principe que les adultes forment une clientèle dont les exigences, en termes de besoins et de didactique, sont tout à fait particulières et différentes de celles des étudiants réguliers; d'où la nécessité d'une structure spécifique ayant pour rôle l'encadrement des adultes dans des activités éducatives élaborées et réalisées exclusivement pour eux, selon un processus administratif et pédagogique approprié. La formule parallèle vise donc principalement à l'autonomie complète de l'éducation des adultes dans l'université.

Quant à la troisième formule, dite "périphérique", elle "se traduit, selon Pineau (1975), par la mise en place, autour d'éléments universitaires centraux, de services aux adultes plus ou moins dépendants" (p. 132). Par leur action, ces services se situent à la périphérie de l'université; ils ont, en effet, pour rôle d'identifier et de préciser les besoins et les attentes du milieu et d'inciter l'université à y répondre par des actions éducatives appropriées. Dans la formule périphérique, l'organisme d'éducation des adultes est donc conçu d'abord comme un organisme "relais": il sert de jonction avec le milieu et même de lieu de confrontation entre les groupes sociaux et l'université.

Un objectif unique. Cette typologie des structures d'éducation des adultes, décrite par Pineau (1975), ne vise pas seulement à cataloguer les approches utilisées par les universités 
ni à donner une perspective globale du développement de l'éducation des adultes en milieu universitaire; elle se situe plutôt dans le cadre d'une analyse des systèmes nouveaux en éducation et cherche essentiellement à montrer la diversité des formules retenues par les universités pour faire de l'éducation des adultes un instrument de transformation du milieu universitaire vers l'éducation permanente. Chacune de ces formules constitue donc, selon sa théorie, une "stratégie de transition pour faire passer un système d'éducation temporaire à une situation d'éducation permanente" (p. 133).

De fait, dans leur effort pour donner suite à la recommandation du Conseil des universités (Québec, 1973) sur l'éducation permanente comme "principe de l'ensemble de notre système d'éducation et en particulier de l'enseignement supérieur" (p. 4), les universités québécoises ont, chacune à sa manière, donné à leurs services d'éducation des adultes les orientations qu'elles croyaient susceptibles d'assurer l'institutionnalisation de l'éducation permanente. Ces changements divers ont donné à l'ensemble des organismes responsables de l'éducation des adultes une allure de mosaique, comme en fait foi la situation de l'éducation des adultes dans les universités décrite lors du Colloque de l'ACDEAULF (1976). A les examiner de près, toutefois, on se rend compte que les diverses approches retenues se ramènent à l'une ou l'autre des trois formules décrites par Pineau et qu'elles ont toutes un but commun: servir de stratégie pour l'implantation de l'éducation permanente.

\section{L'éducation des adultes, une stratégie efficace?}

Il resterait encore, cependant, à développer un instrument qui permette de mesurer objectivement l'efficacité de chacune de ces approches et à procéder aux enquêtes et aux analyses qui s'imposent. Les résultats d'une telle étude sont nécessaires si l'on veut s'assurer que l'éducation des adultes peut effectivement servir de stratégie d'implantation de l'éducation permanente et, le cas échéant, si l'on veut déterminer laquelle des trois approches décrites par Pineau (1975) est la plus avantageuse.

Dans le cas de la formule intégrée, par exemple, il semble que cette stratégie ne puisse contribuer vraiment au développement de l'éducation permanente parce qu'elle comporte des éléments d'auto-destruction qui annulent les résultats escomptés. On sait, en effet, que le regroupement des jeunes et des adultes dans les mêmes activités éducatives empêche l'utilisation d'une approche andragogique véritable et aboutit, à toutes fins pratiques, à une homogénéisation pédagogique orientée vers les jeunes et préjudiciable aux adultes; il s'ensuit que cet effet stratégique de transformation institutionnelle vers l'éducation permanente et, le cas échéant, si l'on veut déterminer laquelle des trois approches décrites par Pineau (1975) est la plus avantageuse.

La formule parallèle offre, par contre, l'avantage de tenir compte des exigences des adultes en termes de besoins et d'approche didactique. Mais, pour favorable qu'elle soit à l'application des principes andragogiques, cette formule n'en comporte pas moins le risque très grand de marginaliser l'éducation des adultes et, par conséquent, de réduire sensiblement, sinon d'annuler, l'effet d'entraînement vers l'éducation permanente qu'elle doit avoir sur l'ensemble de l'université.

Il y a donc lieu de croire, semble-t-il, que l'utilisation de l'éducation des adultes selon la formule intégrée et selon la formule parallèle constituent, pour des raisons propres à chacune, des stratégies peu efficaces pour l'implantation de l'éducation permanente en milieu universitaire: ce sont là, néanmoins, des hypothèses qui mériteraient d'être vérifiées sur la base de données objectives et valables.

Dans le cas de la formule périphérique, nous possédons des données qui, pour être 
limitées aux universités françaises, n'en sont pas moins assez importantes pour nous permettre de mesurer l'efficacité de cette approche comme stratégie d'implantation de l'éducation permanente en milieu universitaire.

\section{LE CAS DES UNIVERSITÉS FRANCAISES}

L'étude qu'il nous a été donné de faire sur la place de la formation continue dans les universités françaises (Ferland, 1979) nous a amené, d'une part, à examiner de près le rôle et le fonctionnement des organismes d'éducation des adultes créés par les universités selon la formule périphérique et, d'autre part, à constater jusqu'à quel point ces organismes ont pu réussir dans leur effort pour amener l'ensemble de l'université vers l'éducation permanente. L'ampleur de cette recherche, en termes de facteurs d'analyse retenus et en termes d'institution recensées, nous autorise à dégager, des données recueillies, certaines leçons sur l'utilisation de l'éducation des adultes comme stratégie d'implantation de l'éducation permanente.

\section{Le rôle des universités françaises en éducation permanente et en formation continue}

Par la loi d'orientation de l'enseignement supérieur (France, 1968), adoptée en novembre 1968 , les universités françaises se sont vu imposer l'obligation de contribuer à l'éducation permanente en travaillant "à la promotion culturelle de la société et par là même à son évolution vers une responsabilité plus grande de chaque homme dans son propre destin" (article 1). A cette fin, les universités doivent, selon cette même loi, accueillir les adultes sur la base de leur aptitude et leur offrir des enseignements adaptés à leurs besoins; elles doivent également faire les recherches pédagogiques nécessaires à l'accomplissement de ces fonctions. Tel est, dans ses grandes lignes, le rôle dévolu aux universités dans le domaine de l'éducation permanente, à charge pour elles de procéder aux adaptations qui s'imposent.

Elles commençaient à peine à s'y consacrer, que survient, en juillet 1971, la loi sur 1'organisation de la formation continue (France, 1971). Selon l'article premier de cette loi, la formation professionnelle continue est constituée "des formations ultérieures destinées aux adultes et aux jeunes déjà engagés dans la vie active ou qui s'y engagent"; elle "fait partie de l'éducation permanente" et, enfin, "elle a pour objet de permettre l'adaptation des travailleurs au changement des techniques et des conditions de travail, de favoriser leur promotion sociale par l'accès aux différents niveaux de la culture et de la qualification professionnelle et leur contribution au développement culturel, économique et social" (article 1).

Notons ici que, par les clientèles auxquelles elle s'adresse (les travailleurs) et par les objectifs qu'elle poursuit (l'adaptation au travail, la promotion sociale et la contribution au développement de la société), la formation continue s'identifie, à toutes fins pratiques, à l'éducation des adultes, telle que définie plus haut: aussi ces deux expressions peuventelles être employées l'une pour l'autre.

La loi met en place un véritable système de formation continue qui considère la formation professionnelle permanente comme une obligation nationale, à laquelle l'Etat et toutes les composantes sociales doivent contribuer et aussi comme un droit pour les travailleurs, à qui il faut donner les moyens nécessaires pour en profiter. Dans ses grandes lignes, ce système comporte trois éléments essentiels: la garantie du congé-formation pour tous les travailleurs, la contribution financière obligatoire des entreprises industrielles et 
commerciales et le mécanisme généralisé de conventions de formation professionnelles entre les entreprises demandeurs et les organismes fournisseurs de formation.

Ce dernier élément présente deux particularités qui méritent d'être soulignées car elles ont une influence sur les conditions dans lesquelles les universités doivent remplir le rôle que la loi leur impose en formation continue. La première de ces particularités est que les actions de formation dispensées doivent faire l'objet d'une négociation avec le prescripteur, tant au point de vue du contenu qu'à celui de la forme et des méthodes didactiques. La seconde particularité réside dans le fait que, contrairement à la situation qui prévaut en formation initiale, les établissements d'enseignement publics et privés ne sont pas les seuls habilités à dispenser la formation continue; ils se trouvent donc en concurrence avec d'autres organismes de formation tels que: les entreprises, les groupes d'entreprises, les associations, les organisations professionnelles, syndicales ou familiales, et les chambres de commerce et d'industrie.

Ainsi donc, dans le domaine de la formation continue, les universités se trouvent placées dans ce que le Ministre de l'Education nationale lui-même appelle un "marché de la formation". Le fonctionnement de ce marché repose sur une triple règle: la négociation des activités éducatives pour adultes, la concurrence entre les différents organismes fournisseurs de formation et l'auto-financement de ces organismes. Dans un tel contexte, qui leur est peu familier, les universités hésitent à assumer les responsabilités que la loi leur impose: elles ne se sentent pas suffisamment compétitives pour conquérir leur part du marché (France, 1972).

\section{La "mission formation continue": une formule périphérique}

Pour aider les universités à prendre leur place sur le marché de la formation et à y jouer le rôle qu'on attend d'elles, l'Etat décide de leur apporter, par un "contrat d'assistance initiale", une aide financière qui prend la forme de ce que l'on a appelé la "mission formation continue".

Définition. La mission formation continue se définit, selon les termes mêmes du Ministère de l'Education (France, 1972), comme une "instance légère d'intervention (...), une cellule légère dirigée par un responsable nommément désigné par le président, disposant de crédits affectés à la conduite des actions" (p. 1224); elle est "chargée d'analyser les besoins de l'environnement, de planifier les ressources de l'université, de négocier des conventions avec les partenaires extérieurs et d'établir un programme cohérent d'actions" (p. 1225). Par ailleurs, dans une note qu'il adressait aux présidents des universités, le Ministre de l'Education (France, 1973) décrivait la mission comme "une masse d'énergie libre, hors structure administrative ou pédagogique afin de leur donner (aux universités) les moyens d'étudier les conditions de réalisation d'une politique cohérente de formation continue" (p. 3).

Structure. Si les missions peuvent être comparées à des masses d'énergie libre, c'est qu'elles n'ont pas de structure véritable. Comme le fait si bien remarquer G. Jobert (1975), "à la différence de l'enseignement secondaire, les universités ne se sont pas vues imposer un modèle de structure (. . .). Chacune, en toute indépendance, a mis en place l'organisation et les procédures qui lui paraissent les plus pertinentes" (p. 102).

De façon générale, cependant, les "chefs de mission" ont été engagés sur une base annuelle, compte tenu du fait que l'aide financière fournie par le gouvernement aux universités était, selon le contrat d'assistance initiale, limitée à une année. Le recrutement s'est fait, le plus souvent, parmi des personnes venant du commerce, de l'entreprise ou de 
l'économie et sensibilisées, par leur formation ou par leur expérience, aux problèmes de l'éducation des adultes: il leur était demandé, en effet, de prospecter un marché et d'élaborer des activités éducatives. Enfin, ces chefs pouvaient organiser leur mission avec les personnels et selon les structures qui leur paraissaient les plus efficaces, compte tenu des budgets dont ils disposaient.

Place et rôle. Par la place qu'elles occupent dans l'université et par le rôle qu'elles ont à jouer, les missions formation continue s'identifient donc parfaitement à ces structures périphériques décrites par G. Pineau (1975) dans son analyse des stratégies d'implantation de l'éducation permanente en milieu universitaire.

Pour ce qui a trait à leur place dans l'université, on peut dire que les missions sont véritablement "hors structures": leur responsable, qui est désigné par le président, ne rend de comptes qu'à lui seul et il dispose d'un budget particulier pour ses actions. Si ce rattachement au président permet "d'éviter un isolement dangereux des activités pour les adultes du reste des préoccupations universitaires", il n'en constitue pas pour autant une intégration véritable dans les structures de gestion ou d'enseignement. La mission se situe donc, en quelque sorte, à la périphérie de l'université.

Par ailleurs, les missions n'ont qu'un rôle d'intervention dans les actions de formation; elles n'en assument pas elles-mêmes l'organisation. Leurs responsabilités, en effet, se limitent essentiellement à la prospection des besoins et des ressources, à l'examen de l'opportunité pour l'université d'entreprendre une action et, le cas échéant, à l'étude des modalités de réalisation de cette action: les missions ne sont pas des structures organisées, chargées de réaliser des actions de formation continue; elles sont plutôt comparables, dans une certaine mesure, à des comités provisoires d'étude. C'est à l'université, dans son ensemble, qu'il incombe d'assurer la réalisation des actions de formation continue: les missions, elles, n'interviennent qu'à titre d'aides dans l'élaboration et dans l'organisation de ces actions.

Mais le rôle des missions ne s'arrête pas là: par leur intervention, elles doivent agir sur l'ensemble de l'université et provoquer ce "renouvellement radical" que suppose la contribution à la politique de formation continue. Bien plus, la nécessaire adaptation de l'université aux exigences de l'éducation permanente devra se faire par le biais de la mission formation continue dans laquelle le gouvernement (France, 1973) voit "une structure particulière de concertation et de réflexion qui permette d'associer à cette action tous ceux qui peuvent y contribuer d'une manière ou d'une autre"' (p. 1229).

Il ne fait donc pas de doute qu'en proposant aux universités de se doter de missions formation continue, le gouvernement français cherchait systématiquement à utiliser l'éducation des adultes comme stratégie d'implantation de l'éducation permanente en milieu universitaire et qu'à cet effet, il optait pour la formule périphérique.

La majorité des universités ont, d'emblée, accepté la formule proposée par le Ministère de l'Education nationale; au cours de l'année universitaire 1972-1973, 67 universités, soit $87 \%$ des 77 établissements d'enseignement supérieur, ont signé avec le gouvernement le contrat d'assistance initiale qui leur permettait de mettre sur pied une mission de formation continue. Diverses raisons ont été invoquées pour expliquer ce succès: la plupart se ramènent à la possibilité, pour l'université, de tirer de la formation continue les éléments stratégiques et les ressources financières utiles à sa transformation vers l'éducation permanente. Les réticences et les résistances qui ont pu surgir ici et là ont été vaincues par l'argument que, même imparfaite, l'approche privilégiée pour l'implantation de l'éducation permanente valait mieux que rien du tout (Chevrolet, 1977). 


\section{Le bilan de l'action exercée par les missions formation continue}

Après cinq ans, il est possible d'établir jusqu'à quel point les missions formation continue ont réussi dans ce double rôle qui leur était confié: d'une part, intégrer la formation continue, c'est-à-dire amener l'ensemble de l'université à répondre aux besoins éducatifs des adultes et, d'autre part, convertir le milieu universitaire aux exigences de l'éducation permanente.

Une enquête, menée au début de 1979 dans 54 universités (sur une possibilité totale de 71 , soit $76 \%$ ) permet en effet de mesurer l'action exercée par les missions formation continue sur l'ensemble de l'université (Ferland, 1979). Cette enquête portait sur certaines pratiques administratives touchant les organismes responsables de la formation continue (v.g. statut, rôle, activités principales, financement) et les personnels attachés à ces organismes (v.g. composition, recrutement, responsabilités) et, sur la base de l'utilisation de ces pratiques, elle visait à établir la place (ou l'importance) que l'université accorde à la formation continue.

Les données recueillies laissent voir que s'il n'est pas possible de porter un jugement clair et définitif sur la place de la formation continue dans l'université, on peut néanmoins déceler, dans l'ensemble des institutions, une forte tendance à considérer, au niveau des principes, la formation continue comme une fonction normale mais à la traiter, dans la pratique, comme une fonction particulière.

Par ailleurs, dans la perspective qui nous intéresse ici, soit l'utilisation de la formation continue (ou de l'éducation des adultes) comme stratégie d'implantation de l'éducation permanente, cette étude montre clairement que les universités, soucieuses de remplir leur rôle en formation continue et de prendre leur place sur le marché de la formation, en sont venues à transformer progressivement leurs missions formation continue, qui avaient été conçues à l'origine comme de simples organismes de sensibilisation, en de véritables "services" occupés principalement à l'organisation et à l'administration d'activités éducatives pour adultes.

Dans l'esprit de tous les intéressés, les missions formation continue devaient constituer un instrument efficace de diffusion de l'éducation permanente en milieu universitaire, comme en fait foi l'insistance avec laquelle on a parlé de leur rôle incitatif à l'intérieur de l'institution. Par le biais de la formation professionnelle continue, la mission devait, croyait-on, sensibiliser toutes les composantes de l'université aux exigences d'une société orientée vers l'éducation permanente. Même la situation du marché, qui était imposée à la formation continue, était supposée, d'une part, amener les universités à s'ouvrir et à s'adapter aux besoins réels du milieu et, d'autre part, leur procurer toutes les ressources financières nécessaires à leur transformation vers l'éducation permanente; mais c'était, dans le premier cas, oublier l'esprit conservateur des universitaires et, dans le second, négliger le fait que le marché était déjà contrôlé en grande partie par les concurrents. Les universités, dans leur ensemble, n'ont donc pas été sensibilisées comme on le souhaitait, un peu en raison des universitaires eux-mêmes peu enclins au changement et beaucoup à cause des ressources financières que le marché n'a pas produites.

Les responsables de la formation continue, quant à eux, n'ont pas ménagé leurs efforts pour intégrer la formation continue dans la structure universitaire. L'enquête a révélé, par exemple, que $75 \%$ des services de formation continue avaient obtenu une reconnaissance officielle de leur Conseil d'université par le biais d'un résolution définissant leur rôle et leur statut. Mais la même enquête a aussi montré que la contrainte de l'auto-financement 
a forcé ces services à organiser eux-mêmes les activités éducatives que les diverses composantes de l'université n'ont pas pris en charge à cause de la lourdeur administrative ou de la résistance des universitaires.

Le développement de la formation continue a donc pris une orientation nettement différente de celle qu'on prévoyait au départ: d'organismes promoteurs, les missions se sont transformées, par la force des choses, en organismes dispensateurs.

Au cours de cette mutation, les services de formation continue ont acquis la double caractéristique de la spécialisation professionnelle et de l'autonomie fonctionnelle. Leurs personnels, en effet, ont été amenés à remplir des fonctions pédagogiques et administratives particulières nettement distinctes de celles qui sont dévolues aux autres universitaires, professeurs ou administrateurs; de plus, les modes de gestion et de financement utilisés par ces services sont tout à fait uniques et ne s'apparentent nullement à ceux qui sont en vigueur dans le reste de l'université.

En somme, les missions formation continue n'ont pas réussi à exercer efficacement l'action qui leur était confiée à l'endroit de l'université. Elles devaient en effet amener l'université à répondre aux besoins identifiés et, de ce fait, la sensibiliser à cette réalité nouvelle qu'est l'éducation permanente; or, l'université n'a pas bougé et les missions ont dû assumer elles-mêmes la mise sur pied et la réalisation des actions de formation que réclamait le milieu, laissant ainsi à l'ensemble de l'université l'illusion qu'elle se transforme vers l'éducation permanente, illusion dont la force croît dans la mesure des succès remportés par les services de formation continue. Ainsi, de périphérique qu'elle était au début, l'éducation des adultes dans les universités françaises est devenue parallèle, voire marginale (Chevrolet, 1977).

\section{Les leçons de l'expérience française}

Bien qu'elle ne s'étende que sur une période de 5 ans environ, l'expérience des universités françaises dans le domaine de l'éducation permanente n'en est pas moins valable, du fait qu'elle présente la double caractéristique de la généralisation et de l'uniformité. Il faut bien remarquer, d'une part, que ce n'est pas seulement une majorité d'universités mais, en réalité, la presque totalité d'entre elles qui se sont lancées, pour ainsi dire, dans l'aventure de la formation continue afin de jouer le rôle précis qu'on leur confiait dans ce domaine et afin de prendre leur place dans celui, plus vaste, de l'éducation permanente. D'autre part, même si l'on a pu observer quelques variations dans les structures organisationnelles adoptées (Jobert, 1975), il n'en reste pas moins que toutes les universités ont, au départ, utilisé la formule privilégiée par l'Etat, soit la mission formation continue, qu'elles ont toutes été placées dans le même contexte de "marché de la formation", basé sur la négociation, l'autofinancement et la concurrence, et qu'elles ont presque toutes commencé à opérer à la même époque, soit entre 1971 et 1973.

L'expérience, puisqu'à bien des points de vue, il s'agit véritablement d'une expérience, s'est déroulée sur une échelle assez vaste, dans des conditions et selon un processus assez uniformes pour prendre valeur de modèle. Quant aux résultats que les organismes de formation continue ont pu obtenir dans leur effort pour implanter le principe de l'éducation permanente, ils sont assez identiques, et par conséquent assez valides et révélateurs, pour donner lieu à un certain nombre de considérations générales. Parmi celles-ci, il en est quatre, principalement, dont la portée est si grande que l'on peut, le plus souvent, en tirer des leçons pour l'ensemble du milieu universitaire.

Une distinction importante. La première de ces constatations est d'ordre conceptuel. 
Le rapprochement que l'on est trop souvent porté à faire entre l'éducation des adultes et l'éducation permanente nous amène à croire, comme on l'a vu plus haut, que ces deux concepts sont équivalents. Or, l'évolution du rôle joué par les missions formation continue nous permet de mesurer concrètement l'écart qui existe entre ces deux concepts et de constater plus particulièrement que l'éducation permanente se situe au niveau des attitudes tandis que l'éducation des adultes (ou la formation continue) se situe à celui de l'action. Cette différence apparaît nettement dans le fait que les missions formation continue, n'ayant pu imprégner à l'ensemble de l'université l'état d'esprit approprié, ont dû ellesmêmes réaliser des activités de formation pour adultes: d'organismes promoteurs qu'elles étaient, les missions sont devenues des organismes dispensateurs de formation.

Cette constatation met en évidence la nécessité pour tout éducateur d'adultes de toujours tenir compte de la différence de niveaux qui existe entre les deux concepts en question, parce qu'elle permet de mieux mesurer la portée des décisions prises au nom de chacun d'eux. Ainsi, par exemple, il importe de savoir si tel geste, que l'on entend poser, se situe dans une optique d'éducation des adultes ou d'éducation permanente: dans le premier cas, en effet, il s'agira d'un geste d'ordre pratique, sporadique ou ponctuel et dont l'effet, par conséquent sera passager parce que situé au niveau de l'action; quant au geste posé dans une optique d'éducation permanente, il aura une portée plus générale et ses effets seront plus significatifs et plus profonds parce qu'il se situe au niveau des attitudes, des comportements; aussi exigera-t-il plus d'attention et plus de doigté.

Une autonomie nécessaire. La deuxième constatation que l'expérience française nous amène à faire est que l'éducation des adultes a besoin, pour se développer, d'une certaine autonomie. Les missions formation continue ont rempli efficacement la première moitié de leur tâche: elles ont soigneusement prospecté le marché, y ont décelé des besoins de formation continue et les ont transmis à l'université. Mais, en général, les universités n'ont pas bougé et les missions formation continue ont dû se transformer en services et dispenser elles-mêmes de l'enseignement.

On est porté à attribuer cet immobilisme des universités soit à la lenteur et à la complexité de la "machine" administrative, soit à la résistance au changement que les universitaires ont, paraît-il, développée d'un façon particulière (Passeron, 1970). Mais, quand on scrute un peu les motifs allégués par ceux qui refusent de contribuer à des actions de formation continue, on découvre qu'ils ont pour véritable fondement la nécessité d'adopter une approche pédagogique et un processus administratif bien différents de ceux qu'on utilise en formation initiale.

L'éducation des adultes ne peut s'accommoder entièrement des règles de fonctionnement qui régissent l'éducation des jeunes: c'est ce que démontre clairement le fait que, dans l'ensemble, les universités françaises n'ont pu assumer entièrement et pleinement leurs responsabilités en formation continue et qu'elles les ont plutôt confiées à des organismes spécialisés relativement autonomes sur les plans pédagogique et administratif. Ce phénomène, que l'on qualifie parfois de "marginalisation de l'éducation des adultes" apparaît sur le plan didactique comme une manifestation de l'originalité de l'approche andragogique; sur le plan organisationnel, il met en évidence le problème du statut et du rôle particulier que l'université devra attribuer à l'organisme et au personnel qui sont responsables de l'éducation des adultes.

Un marché à conquérir. L'expérience vécue par les universités françaises met un troisième fait en évidence: les universités n'ont pas, dans le domaine de la formation continue, le quasi-monopole qu'elles détiennent en formation initiale car elles sont, ici, dans une situa- 
tion de concurrence. Le "marché de la formation", dans lequel les universités françaises sont placées, n'est pas, à proprement parler, une création de la loi de juillet 1971; il existait auparavant et la loi n'a fait que le confirmer et, en quelque sorte, le régulariser.

Or, cette situation qui prévaut en formation continue, n'est pas particulière à la France; elle existe dans d'autres pays, sans doute à un degré de développement moindre, mais elle n'en est pas moins réelle. A mesure que la nécessité de l'éducation permanente se fait sentir dans l'ensemble de la société et à mesure, surtout, que la formation professionnelle continue apparaît comme un investissement pour l'industrie et le commerce, il se crée, parallèlement aux institutions publiques d'enseignement, un ensemble d'organismes privés, spécialisés dans la formation des adultes et opérant selon le principe de la rentabilité financière. Ces organismes se posent en concurrents aux institutions traditionnelles d'enseignement et leur présence a pour effet de transformer l'éducation des adultes en un véritable marché de la formation continue comparable à celui de la France.

Cette situation entraîne, pour les universités, la nécessité de développer des comportements concurrentiels pour aller rejoindre la clientèle de la formation continue. A titre de service public, il leur faut, en effet, être en mesure de répondre aux besoins éducatifs du milieu et, pour ce faire, elles doivent prendre leur part du marché. Il leur reste ensuite à déterminer jusqu'à quel point elles peuvent aller sur cette voie sans risquer de devenir des entreprises commerciales.

Une stratégie peu efficace. Enfin, la quatrième constatation importante qu'on peut tirer de l'expérience française concerne l'utilisation de l'éducation des adultes comme stratégie d'implantation de l'éducation permanente. Les missions formation continue devaient, par l'intégration de l'éducation des adultes à l'ensemble de l'université, amener progressivement celle-ci à adopter le principe de l'éducation permanente; mais elles n'ont pu réussir ni à faire entrer véritablement l'éducation des adultes dans l'université ni, par conséquent, à faire accepter le principe de l'éducation permanente. Sur le plan organisationnel, les missions constituaient, comme on l'a vu, des applications du modèle périphérique selon la typologie de Pineau (1975). Que ces missions n'aient pas réussi à convertir l'université à l'éducation permanente nous porte à croire que l'approche périphérique ne constitue pas une stratégie efficace d'implantation de l'éducation permanente en milieu universitaire; cela nous incite, également, à rechercher des moyens de vérifier l'efficacité des deux autres approches, soit l'approche intégrée et l'approche parallèle.

Par ailleurs, que les missions formation continue, qui, à l'origine, étaient des structures périphériques, aient non seulement échoué à faire assumer l'éducation des adultes par l'ensemble de l'université, mais encore se soient elles-mêmes transformées en des services parallèles à l'université, cela nous amène à nous demander si l'éducation des adultes constitue, de quelque façon que ce soit, la meilleure stratégie d'implantation de l'éducation permanente en milieu universitaire. Il faut, vraisemblablement, cesser de croire que c'est uniquement - et même principalement - par l'éducation des adultes que va s'instaurer l'éducation permanente.

\section{CONCLUSION}

Aussi paradoxal que cela puisse paraître, il semble bien que ce soit par l'éducation des jeunes que l'université en viendra à s'adapter à l'éducation permanente. Les jeunes qui arrivent à l'université présentent de plus en plus certaines caractéristiques qui leur sont particulières et que n'avaient pas toujours leurs prédécesseurs: ils ont acquis beaucoup de 
connaissances en dehors du système éducatif, par des moyens techniques peu traditionnels (v.g. la télévision et le voyage) et sur des sujets divers et peu scolaires. Ils sont rompus à certaines formes nouvelles d'apprentissage et ils affichent, à l'endroit de leur propre éducation, une attitude telle qu'ils n'hésitent pas à en interrompre parfois le processus, comme le montre le phénomène des "stop-outs".

Or, ce sont là, à vrai dire, les caractéristiques d'une éducation permanente authentique, c'est-à-dire, d'une éducation qui considère que le développement de l'individu peut s'accomplir en dehors du système scolaire, grâce à l'acquisition de connaissances non scolaires, par des moyens autres que l'enseignement traditionnel et selon un rythme personnel.

Cette éducation-là, qui est la vraie éducation permanente, ce sont vraisemblablement les générations futures de jeunes qui vont l'imposer à l'université; l'éducation des adultes, pour sa part, pourra contribuer à ce mouvement, mais elle ne sera pas l'agent unique, ni même l'agent principal de l'implantation de l'éducation permanente en milieu universitaire.

\section{REFERENCES}

ACDEAULF. L'avenir de l'éducation des adultes dans les universités canadiennes de langue française. Actes du Séminaire de l'Association Canadienne des Dirigeants de l'Education des Adultes des Universités de Langue Française, Mont Orford, 26-28 mai 1976.

Belorgey, J.M. Formation continue: un premier bilan. Projet, fév. 1974, 82, p. 183.

Chevrolet, D. L'université et la formation continue: signe et sens d'une situation de l'éducation. Tournai: Casterman, (Orientations/E3), 1977, 193 p.

Conseil de l'Europe. Education permanente: un recueil d'études commanditées par le Conseil de la Coopération culturelle. Strasbourg: Conseil de l'Europe, 1970, pp. 78-79.

Ferland, M. Les pratiques administratives et la place de la formation continue à l'université. Thèse pour le doctorat d'université en administration des entreprises, Institut de gestion de Rennes, Université de Rennes, 1979, (non publiée).

France. Bulletin officiel de 1'Education nationale. Circulaire No 72-187 du 26 avril 1972: Modalités d'application de la loi No 71.575 du 16 juillet 1971 portant organisation de la formation professionnelle continue dans le cadre de l'éducation permanente (signé R. Vatier), B.O.E.N., 4 mai 1972 , No 18 , p. 1219-1231.

France. Journal officiel de ta République française. Loi No $68-878$ du 12 novembre 1968 d'orientation de l'enseignement supérieur, (J.O. du 13 nov. 1968).

France. Journal officiel de la République française. Loi No $71-575$ du 16 juillet 1971 portant organisation de la formation professionnelle continue dans le cadre de l'éducation permanente, (J.O. du 17 juillet 1971).

France. Ministère de l'éducation nationale. Instruction du 28 juin 1973 présentant le contrat de développement, (non publié).

Houle, Cyril O. Adult Education. In Encyclopedia of Educational Research (4th Ed.). London: Collier-Macmillan, 1969, pp. 51-57.

Jobert, G. Politique et stratégies d'implantation de la formation continue en milieu universitaire. Connexions politiques et expériences de formation, déc. 1975, 16, pp. 93-103.

Passeron, J.C. La forme universitaire de résistance à l'innovation. Les Cahiers del'AUPELF (Association des universités partiellement ou entièrement de langue française), sept. 1970, pp. 125-131.

Pineau, G. Une stratégie périphérique de changement organisationnel en éducation. Education permanente, janv.fév. 1975, 27, pp. 129-145.

Québec. Conseil des universités. Objectifs généraux de l'enseignement supérieur et grandes orientations des établissements (Cahier II). Québec, 22 fév. 1973. 